\title{
The effect of sleep on men's health
}

\author{
Taylor P. Kohn ${ }^{1}$, Jaden R. Kohn ${ }^{2}$, Nora M. Haney ${ }^{1}$, Alexander W. Pastuszak ${ }^{3}$, Larry I. Lipshultz ${ }^{4,5}$ \\ ${ }^{1}$ The James Buchanan Brady Urological Institute and Department of Urology, ${ }^{2}$ Department of Gynecology and Obstetrics, Johns Hopkins University \\ School of Medicine, Baltimore, MD, USA; ${ }^{3}$ Division of Urology, Department of Surgery, University of Utah School of Medicine, Salt Lake City, \\ UT, USA; ${ }^{4}$ Scott Department of Urology, ${ }^{5}$ Center for Reproductive Medicine, Baylor College of Medicine, Houston, TX, USA \\ Contributions: (I) Conception and design: AW Pastuszak, LI Lipshultz; (II) Administrative support: TP Kohn, LI Lipshultz, JR Kohn, NM Haney; (III) \\ Provision of study materials or patients: None; (IV) Collection and assembly of data: TP Kohn, NM Haney; (V) Data analysis and interpretation: All \\ authors; (VI) Manuscript writing: All authors; (VII) Final approval of manuscript: All authors. \\ Correspondence to: Larry I. Lipshultz, MD. Professor, Chief, Division of Male Reproductive Medicine and Surgery, Scott Department of Urology, \\ Baylor College of Medicine, 6624 Fannin Street, \#1700, Houston, TX 77030, USA. Email: larryl@bcm.edu.
}

\begin{abstract}
Sleep has increasingly been found to play a role in the overall health of an individual, but sleep quality has also been decreasing with the invasion of technology into the bedroom, "always-on" lifestyles, and increasing demands on one's time when awake. We have herein reviewed the literature to assess the impact of sleep on erectile dysfunction, lower urinary tract symptoms, hypogonadal symptoms, low testosterone, and male infertility. We find that erectile dysfunction, lower urinary tract symptoms, and hypogonadal symptoms all have a linear relationship with sleep, as worse symptoms occur with poorer sleep. Male infertility, interestingly, has an inverse U-shaped relation to sleep in which men with too little and too much sleep seem to be more at risk for infertility than those with 7-8 hours of sleep. Finally, the literature has not demonstrated a significant clinical relationship between hypogonadal symptoms or testosterone levels and sleep. Overall, a large number of men experience poor quality sleep. Given the impact that poor sleep can have on general health and men's health, in particular, screening for poor sleep quality and recommending interventions to improve sleep are becoming imperative during clinical evaluation and treatment.
\end{abstract}

Keywords: Health; men's; sleep; erectile dysfunction; lower urinary tract symptoms

Submitted Sep 04, 2019. Accepted for publication Nov 11, 2019.

doi: 10.21037/tau.2019.11.07

View this article at: http://dx.doi.org/10.21037/tau.2019.11.07

\section{Introduction}

Sleep has long been known to play a critical role in health. Over the past several decades, a large number of studies have demonstrated that poor sleep is associated with worse health outcomes (1). The American Academy of Sleep Medicine and the Sleep Research Society suggest that 7 to 9 hours of sleep are required for optimal health in adults (2). However, a large proportion of American adults fail to achieve this 7-hour minimum (3). A 2012 survey demonstrated that $29.2 \%$ of men achieved less than 6 hours of sleep on average per night (4). The Institute of Medicine estimates that 50-70 million Americans have a chronic sleep disorder (5). This epidemic of chronic sleep deprivation can largely be traced back to broad societal changes including increasing dependence on technology, longer work hours, and poor sleep hygiene (6,7). Additionally, nonstandard shift work (which affects approximately 21 million Americans) further disrupts circadian rhythms and impairs sleep quality (8).

The relationship between sleep and health is bidirectional. Numerous medical conditions such as sleep apnea, restless leg syndrome, insomnia, hypogonadism, and depression can all result in impaired sleep, but often sleep can exacerbate the symptoms of these conditions (6). Conversely, many studies have demonstrated that poor sleep and short sleep duration are associated with numerous health conditions, including coronary heart disease, worsened diabetes and hypertension, stroke, myocardial infarctions, and more $(6,8,9)$. The effects of poor sleep can significantly impact 


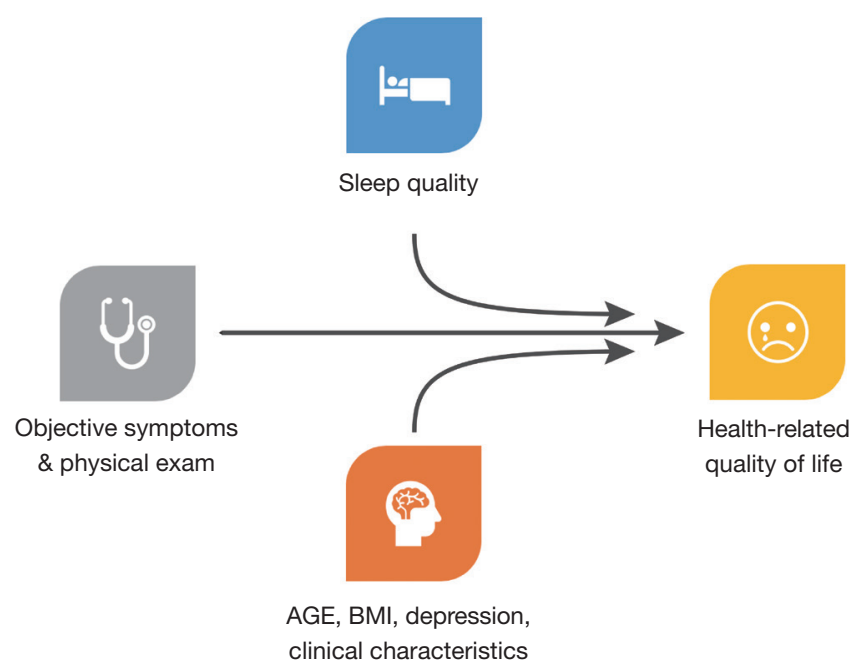

Figure 1 Sleep functioning as a moderator of clinical symptoms.

not only biologic responses but can also alter the way that patients perceive their symptoms (10) (Figure 1).

In this review, we will assess the impact of sleep on common conditions affecting men's health, including erectile dysfunction, lower urinary tract symptoms, hypogonadal symptoms, low testosterone, and male infertility.

\section{Methods}

A literature review was performed in MEDLINE and EMBASE using terms related to erectile function, benign prostatic hyperplasia, lower urinary tract symptoms, bladder outlet obstruction, hypogonadism, testosterone, male infertility, sleep, insomnia, obstructive sleep apnea, shift work, and shift work sleep disorder. All article types were included. Searches were performed by limiting the language only to English.

\section{Sleep and erectile function}

Longitudinal studies have found that nearly $50 \%$ of men experience some degree of erectile dysfunction (ED) (11). The impact of sleep on erectile function has been explored in several recent studies. In a cross-sectional analysis of 2,676 community-dwelling men, aged 67 years or older, Bozorgmehri et al. found that men with nocturnal hypoxemia had higher risks of experiencing moderate or complete erectile dysfunction (OR 1.39, 95\% CI: 1.00-1.92) (12). This same trend is evident in a systematic review and meta- analysis assessing the impact of obstructive sleep apnea (OSA) on erectile function. Across ten studies, men without sleep apnea were much less likely to experience ED (OR 0.45, 95\% CI: 0.18-0.71) (13).

Given this strong association between obstructive sleep apnea and erectile dysfunction, Melehan et al. performed a randomized sham-controlled trial to determine whether initiation of continuous positive airway pressure (CPAP) would improve erectile function in men with ED and OSA. A total of 61 men were randomized to CPAP or sham CPAP for 12 weeks. While men randomized to CPAP experienced an increase in sleep-related erections and overall sexual satisfaction, no change was seen in erectile function, as assessed by the International Index of Erectile Function (IIEF). When stratified by men who were adherent to CPAP use (defined as the use of CPAP for $>4$ hours per night) and those who were not adherent to CPAP use, men with adherent use experienced a significant improvement of erectile function (14). Melehan $e t$ al. randomized men to receive Vardenafil or placebo as part of their two-by-two factorial analysis, and showed that a PDE-5 inhibitor did not improve erectile function in men with OSA (14). Thus appropriate use of CPAP can reverse the impact of OSA on erectile function, perhaps beyond that achievable by simply prescribing PDE5i. McBride et al. found that $27.4 \%$ of men presenting to an academic men's health clinic were at high risk for OSA, but of those referred for polysomnography testing, only $50 \%$ of men ever follow up on this referral (15). The evidence certainly suggests that OSA significantly impacts erectile function, that men with OSA may not respond to first line treatment for ED with PDE5i, and that correcting underlying sleep impairment may result in a more meaningful improvement in erectile function.

OSA is not the only sleep disruptor that has been shown to impair erectile function. Another population that experiences significant sleep deprivation is men who work non-standard shifts. In a cross-sectional study of 182 men who worked non-standard shifts (work schedules that begin before 7 am or after 6 pm, regularly extend outside of a 7 am to $6 \mathrm{pm}$ frame, or that frequently rotate in schedule). Pastuszak et al. showed that men who were "very dissatisfied" with sleep had significantly lower IIEF scores $(\mathrm{P}=0.001)$ (16). In a follow up study, Rodriguez et al. demonstrated that in a cross-sectional analysis of 802 men that, while shift work alone did not predict significantly worse erectile function $(\mathrm{P}=0.31)$, men who had shift work sleep disorder had IIEF scores that were 2.8 points lower than men who worked similar non-standard shifts but 
who did not have shift work sleep disorder $(\mathrm{P}<0.01)$. Shift work sleep disorder is a circadian rhythm disruption sleep disorder characterized by at least one month of excessive sleepiness, insomnia, and impairment of social and occupational activities attributed to a persistent shift work schedule (17). Thus, like OSA, shift work that significantly impairs sleep (such as shift work sleep disorder) can result in worsened erectile function.

While formal diagnoses of OSA and shift work sleep have been shown to impact erectile function, impaired overall sleep quality and insomnia can also cause a significant impact on erectile function. In a cross-sectional survey assessing the impact of sleep on sex using the insomnia severity index and IIEF, Seehuus et al. found that men at high risk for insomnia had significantly lower IIEF scores than their counterparts who were at low risk for insomnia (18). This correlation between insomnia and erectile dysfunction has been confirmed by several other studies $(19,20)$. The evidence linking sleep and erectile dysfunction are growing, and assessment of sleep during an erectile function workup can be easily performed with validated sleep questionnaires such as the Pittsburgh Sleep Quality Index (PSQI). While no study has assessed the impact of PDE5i on men with poor sleep and ED, the correction of poor sleep alone may be enough to lead to clinically improved erectile function.

\section{Sleep and lower urinary tract symptoms}

Another commonly experienced condition in the aging male is lower urinary tract symptoms (LUTS) associated with benign prostatic hypertrophy. These clusters of symptoms include incomplete bladder emptying, poor stream, straining to urinate, hesitancy, frequency of urination, and nocturia. Given that men often have the urge to urinate overnight, it is not surprising that this constant sleep disturbance can affect the overall quality of sleep. In a survey of 5,335 men with a diagnosis of LUTS, patients with nocturia reported that they were "always" or "usually" tired significantly more often than men who only reported daytime urinary problems (21). This survey also found that only $13 \%$ of men with nocturia reported continuous sleep periods of more than 2-3 hours (21). Thus clearly, nocturia within the context of lower urinary tract symptoms affects sleep significantly.

Evidence, however, is growing that poor sleep itself may lead to the worse experience of LUTS. It has long been known that the size of the prostate and the severity of LUTS are not well correlated; rather, it may be that the subjective experience of LUTS can be affected by other factors. Men with OSA were significantly more likely to have moderate and severe lower urinary tract symptoms compared with men who do not have OSA. Interestingly, the severity of OSA correlated the most strongly with storage symptoms: daytime frequency, urgency, and frequency of nocturia (22). In multivariate linear regression, Bates et al. showed that men at high risk for OSA had IPSS scores that were 3.2 points higher than men without OSA; men who reported "very bad sleep" had IPSS scores 7 points higher than men with "fairly good sleep" and 10 points higher than men with "very good sleep" (23).

Fortunately for men with OSA, continuous positive airway pressure (CPAP) devices can restore the quality of sleep and reverse the detrimental impact of OSA on health (24). Specific to the aims of this paper, studies have demonstrated that CPAP use in men with LUTS can improve these symptoms. Fernandez-Pello et al. found that for men with LUTS and OSA who used CPAP for 1 year, IPSS scores decreased by 3.6 points, and bladder compliance significantly increased from 97 to $200 \mathrm{~mL} / \mathrm{cm}$ of $\mathrm{H}_{2} \mathrm{O}$ (25). Further, Irer et al. found that when with men with OSA and LUTS consistently used CPAP, nighttime urine volumes decreased from 547 to $96 \mathrm{cc}(\mathrm{P}<0.001)$ and the frequency of nocturia decreased from 2.1 to 0.5 bathroom trips at night (22). Fortunately, the presence of LUTS has not been found to decrease adherence to CPAP use when compared to men without LUTS (26).

OSA is not the only sleep disturbance that has been demonstrated to impact LUTS. Shift work has also been shown to hurt LUTS. Scovell et al. found that, in men who performed nonstandard shifts, those men who have increased difficulty falling asleep, staying asleep, or falling back asleep reported more severe LUTS than men without difficulty falling asleep (27). Shift workers diagnosed with shift work sleep disorder had significantly worse IPSS scores (3.74 points higher) compared with shift workers without shift work sleep disorder $(\mathrm{P}<0.0001)$ when controlling for age, benign prostatic hyperplasia, comorbidities, and testosterone (28). Overall, sleep disturbances brought on by shift work appear to impact LUTS.

Insomnia has also been related to worsened LUTS (23). Unlike OSA and shift work sleep disorder, insomnia has an FDA-approved medication-ramelteon-which is a melatonin receptor agonist. Shimizu et al. found that in men with insomnia and LUTS treated with ramelteon, IPSS scores significantly declined from 11.4 to 9.4 $(\mathrm{P}=0.0013)$, although the quality of life did not improve as 
dramatically (3.6 to $3.1, \mathrm{P}=0.0009$ ) (29). This early study may indicate that insomnia associated with LUTS could be ameliorated with ramelteon and that more studies will have to investigate this subpopulation further.

\section{Sleep and hypogonadism}

Unlike the ED, as mentioned earlier and LUTS, the relationship between hypogonadism and sleep has not been as thoroughly explored. The AUA guidelines define hypogonadism as (I) a testosterone level $<300 \mathrm{ng} / \mathrm{dL}$ on at least two AM serum total testosterone measurements and (II) the presence of select symptoms or signs including erectile dysfunction, decreased libido, lethargy, and/ or sleep disturbances (30). Patel et al. found a linear relationship between testosterone levels and duration of sleep within the 2011-2012 National Health and Nutrition Examination Survey. On multivariate regression controlling for age, BMI, and comorbidities, authors demonstrate that for every less hour of sleep, testosterone levels decreased by $5.9 \mathrm{ng} / \mathrm{dL}$ (3). While this represented a statistically significant difference $(\mathrm{P}<0.01)$, clinically, the relationship is less clear as a $5 \mathrm{ng} / \mathrm{dL}$ decrease may not be clinically significant. Pastuszak et al. examined this same relationship in shift workers and found no significant difference in mean serum testosterone, free testosterone, dehydroepiandrosterone, follicle-stimulating hormone, or luteinizing hormone levels (16). Testosterone levels also did not appear to be affected when examining men with shift work sleep disorder, as authors found a non-significant difference in testosterone levels when comparing shift workers with and without shift work sleep disorder (31). $\backslash$ Nor does it appear that OSA significantly impacts testosterone levels (32). Thus sleep appears to have little or no significant impact on testosterone levels.

While testosterone levels do not appear to be impacted by sleep, the severity of symptoms associated with hypogonadism certainly does appear to be affected by sleep. We have already demonstrated how poor sleep is associated with erectile dysfunction, but other symptoms of hypogonadism, such as poor libido, poor energy, and decreased strength, can also be affected by poor sleep quality. These symptoms can be assessed with the validated questionnaire-Androgen Deficiency in Aging Males (ADAM) — as well as several other questionnaires (33). One study of 144 men demonstrated that those with positive ADAM had significantly worse sleep $(\mathrm{P}<0.001)$ compared with men with negative ADAM (34). While Pastuszak et al. found no significant difference in testosterone levels, they did find a significant difference in ADAM scores, as men with worse sleep had higher ADAM scores $(\mathrm{P}<0.05)(16)$. This relationship was even more accentuated when examining men with shift work sleep disorder-those men with shift work sleep disorder had more significant symptoms associated with hypogonadism despite there being no difference in serum testosterone levels compared with men without shift work sleep disorder (31). Thus, clearly, the literature suggests that sleep negatively impacts the symptoms associated with hypogonadism, even if testosterone levels are relatively unchanged. This phenomenon may prove useful in a certain subgroup for the management of hypogonadal symptoms. As a result of the new AUA guidelines defining hypogonadism as a testosterone level $<300 \mathrm{ng} / \mathrm{dL}$, men with severe symptoms of hypogonadism who have testosterone levels above $300 \mathrm{ng} / \mathrm{dL}$ do not meet the guidelines for testosterone replacement therapy. In this group, it may be that behavioral modifications, such as improving sleep quality, losing weight, and eating healthier, may improve the symptoms that they are experiencing without testosterone supplementation.

\section{Sleep and male fertility}

Infertility is another common urologic condition that is affecting an increasing number of men. Recent population studies have demonstrated that sperm counts have been decreasing, with one meta-analysis demonstrating a $50-60 \%$ decrease over the past 40 years $(35-37)$. The relationship between sleep and fertility, however, is not as linear as it is with erectile dysfunction, LUTS, and hypogonadal symptoms-in which cases, less sleep or worse sleep quality results in worsening symptoms. In the case of fertility, the evidence suggests that an inverted U-shaped relationship exists in which both less sleep and excessive sleep are associated with worse fertility outcomes. In a large, prospective, web-based cohort study, Wise et al. enrolled 1,176 couples who had been attempting to conceive for up to 6 cycles. Comparing 8 hours of sleep per night, men who sleep $<6$ hours had fecundability ratios (FR) of 0.62 (95\% CI: 0.45-0.87), men who sleep 6 hours per night had an FR of 1.06 (95\% CI: 0.87-1.30), men who sleep 7 hours per night had FR of 0.97 (95\% CI: 0.81-1.17), and finally those who sleep $\geq 9$ hours per night had FR of 0.73 (95\% CI: $0.46-1.15)$. This study suggests that men at the extremes of sleep duration may have impaired fecundity (38). 
This is not the only study to suggest this inverted U-shaped relationship. Kohn et al. found that in a cohort of 198 infertile men, those whom self-reported moderate sleep difficulty had sperm concentrations that were 15.4 million sperm $/ \mathrm{mL}$ greater than men who reported no sleep difficulty and 4.72 million sperm $/ \mathrm{mL}$ greater than those who reported severe sleep difficulty $(\mathrm{P}=0.018)$.

No difference in semen volume, sperm motility, LH, or FSH levels were observed (39). Two other studies have also reported an inverted U-shaped relationship between sleep and sperm concentration-one assessing 796 Chinese college students and another assessing 953 Danish military recruits $(40,41)$. This distribution in semen parameters and fertility outcomes may be explained by circadian disruption. Melatonin, a key circadian hormone, is also a potent antioxidant that has also been found to play a role in the testicle (42). Testicular biopsies in idiopathic infertile men have found an inverse relationship between testicular melatonin concentrations and inflammatory factors in the testes such as TNF $\alpha$, IL1 $\beta$, and COX2. Authors also found that increased testicular melatonin concentrations are correlated with increased expression of antioxidant enzymes (43). It may be that circadian disruption from either too little or too much sleep could affect the oxidation state of the testicle, tipping spermatogenesis one way or the other.

No studies have yet assessed the impact of OSA on fertility. This relationship may be a potential area to explore as addressing undiagnosed OSA in men has been linked with significant improvement in overall health risks, which may spill over into fertility parameters.

Lastly, shift work has been suggested to impair spermatogenesis. In a study of 522 men, El-Helaly et al. reported that men who performed shift work had higher odds of experiencing infertility (OR 3.60, 95\% CI: 1.1211.57 ) (44). However, this relationship has not been seen consistently. A large European multi-center study found no effect on fecundity in men who perform shift work (OR 1.46, 95\% CI: 0.89-2.40) (45). Finally, no study has yet assessed the impact of shift work sleep disorder and insomnia on male fertility.

\section{Potential treatments and screening for sleep deficiency}

Given the increasing evidence of the impact of poor sleep on health, more studies are beginning to assess potential treatments to ameliorate poor sleep and its downstream effects. One of the simplest approaches is to improve sleep hygiene. Sleep hygiene includes a variety of practices and habits that contribute to the quality of nighttime sleep and daytime alertness. In recent years, sleep hygiene has suffered from the invasion of technology into the bedroom via portable screens and the "always-on-the-go" mentality (7). Poor sleep hygiene can be overcome with simple daily modifications such as avoiding daytime naps, avoiding caffeine and nicotine close to bedtime, regular exercise, increased daytime exposure to sunlight, and limiting electronic screen light before bedtime (46). Only two studies have assessed whether sleep hygiene is associated with urologic outcomes. Bates et al. demonstrated that men with better sleep hygiene had higher IPSS scores when compared with men with poor sleep hygiene (IPSS difference 2.7, $\mathrm{P}<0.01)$ (23). Additionally, Kohn et al. found that men with better sleep hygiene had statistically higher IIEF-EF scores compared with men with poor sleep hygiene (IIEF-EF difference $-1.4, \mathrm{P}<0.05$ ) (47). Improving sleep hygiene is a no-cost intervention that may help to improve a patient's perspective of their symptoms and lead to a better quality of life.

As detailed above, sleep apnea is important to diagnosis, and sleep apnea can be treated with CPAP intervention. Several questionnaires exist to identify those at high risk for sleep apnea; one of the most simple and easily implemented questionnaires is the STOP-Bang questionnaire (48). This 8 -question validated screening tool can be easily given to any patient undergoing evaluation in a clinic. The tool assesses a patient for snoring, daytime tiredness, observed apneic events, hypertension, BMI, age, neck circumference, and gender. Patients with a positive screening tool should be referred for sleep polysomnography. Walia et al. demonstrated that $39 \%$ of men presenting to a men's health clinic were at "high risk" for sleep apnea (49)—thus, men presenting to a men's health clinic merit universal screening for OSA as they are at elevated risk. However, sleep apnea should not be the only inquiry for providers. A simple question of "how are you sleeping?" during clinical evaluation may help to unlock other sleep deficiencies. In patients who provide negative or ambiguous responses, physicians can further define these sleeping deficiencies with the Pittsburgh Sleep Quality Index (PSQI). This 19-question index takes approximately five to ten minutes to complete, but provides seven "component" scores: subjective sleep quality, sleep duration, sleep disturbances, sleep latency, use of sleeping medication, habitual sleep efficiency, and daytime dysfunction (50). Patients with 
abnormal subcomponents can be referred to primary care physicians or sleep specialists if the PSQI reveals deficits. In patients with demonstrated poor sleep hygiene on the PSQI, simple sleep hygiene education handouts may help patients to improve their sleep hygiene, which could lead to an improvement in their presenting symptoms (51). Inquiry into patients' sleep is slowly being incorporated into clinical practice, but this should be assessed with every new patient presenting for evaluation at a men's health clinic.

There are many future avenues for research within the subfield of sleep and men's health. One hypothesis is that improving sleep may improve disorders such as hypogonadal symptoms, LUTS, erectile dysfunction, or fertility—but this has not been yet explored. A further interesting inquiry would be to assess whether patients with ED or LUTS who have sleep deficiencies respond equally to standard-of-care pharmaceutical intervention when compared to matched men without sleep deficiencies. Perhaps men with poor sleep do not respond to these pharmaceutical interventions with similar magnitude of improvement without also addressing underlying sleep issues. Finally, while technology may be eroding sleep habits, perhaps it can also be a means for improving sleep. Recently, several developers have created patient-facing smartphone apps that provide cognitive behavioral therapy for insomnia (CBT-I) (52). CBT-I is one of the most effective methods for treating insomnia and poor sleep (51). Bringing this intervention into a patient's home with innovative technology may be able to broaden the reach of CBT-I and could be an interesting avenue for researchers to pursue as a potential treatment for the conditions mentioned above. While the study of sleep has begun to yield troubling findings, the investigation of the role of sleep within urology is just beginning and may be an important risk factor that significantly modifies patients' urologic symptoms.

\section{Conclusions}

In this review of the literature, we investigated the effect of sleep on men's health conditions, including ED, LUTS, hypogonadism, and male infertility. In all cases, poor sleep has been demonstrated to impact the symptoms that these men experience negatively. Sleep deficiency is a common but often missed diagnosis in men presenting for urologic care. Sleep wellness is an important and modifiable risk factor for providers to identify in men as several interventions can improve sleep quality and possibly alleviate some presenting symptoms.

\section{Acknowledgments}

Funding: None.

\section{Footnote}

Provenance and Peer Review: This article was commissioned by the Guest Editors (Larry I. Lipshultz, Alexander W. Pastuszak) for the focused issue "Contemporary Issues and Controversies in Men's Health" published in Translational Andrology and Urology. The article was sent for external peer review organized by the Guest Editors and the editorial office.

Conflicts of Interest: The focused issue "Contemporary Issues and Controversies in Men's Health" was commissioned by the editorial office without any funding or sponsorship. LIL serves as an unpaid editorial board member of Translational Andrology and Urology from Nov 2019 to Oct 2021. LIL and AWP served as the unpaid Guest Editors of the focused issue. The other authors have no conflicts of interest to declare.

Ethical Statement: The authors are accountable for all aspects of the work in ensuring that questions related to the accuracy or integrity of any part of the work are appropriately investigated and resolved.

Open Access Statement: This is an Open Access article distributed in accordance with the Creative Commons Attribution-NonCommercial-NoDerivs 4.0 International License (CC BY-NC-ND 4.0), which permits the noncommercial replication and distribution of the article with the strict proviso that no changes or edits are made and the original work is properly cited (including links to both the formal publication through the relevant DOI and the license). See: https://creativecommons.org/licenses/by-nc$\mathrm{nd} / 4.0 /$.

\section{References}

1. Deng N, Haney NM, Kohn TP, et al. The Effect of Shift Work on Urogenital Disease: a Systematic Review. Curr Urol Rep 2018;19:57.

2. Watson NF, Badr MS, Belenky G, et al. Recommended Amount of Sleep for a Healthy Adult: A Joint Consensus Statement of the American Academy of Sleep Medicine and Sleep Research Society. Sleep 2015;38:843-4. 
3. Patel P, Shiff B, Kohn TP, et al. Impaired sleep is associated with low testosterone in US adult males: results from the National Health and Nutrition Examination Survey. World J Urol 2019;37:1449-53.

4. Ford ES, Cunningham TJ, Croft JB. Trends in SelfReported Sleep Duration among US Adults from 1985 to 2012. Sleep 2015;38:829-32.

5. Institute of Medicine Committee on Sleep M, Research. The National Academies Collection: Reports funded by National Institutes of Health. In: Colten HR, Altevogt BM, editors. Sleep Disorders and Sleep Deprivation: An Unmet Public Health Problem. Washington (DC): National Academies Press (US). National Academy of Sciences.; 2006.

6. Luyster FS, Strollo PJ, Jr., Zee PC, et al. Sleep: a health imperative. Sleep 2012;35:727-34.

7. Gamble AL, D'Rozario AL, Bartlett DJ, et al. Adolescent sleep patterns and night-time technology use: results of the Australian Broadcasting Corporation's Big Sleep Survey. PLoS One 2014;9:e111700.

8. Deng N, Kohn TP, Lipshultz LI, et al. The Relationship Between Shift Work and Men's Health. Sex Med Rev 2018;6:446-56.

9. Matricciani L, Bin YS, Lallukka T, et al. Past, present, and future: trends in sleep duration and implications for public health. Sleep Health 2017;3:317-23.

10. Lautenbacher S, Kundermann B, Krieg JC. Sleep deprivation and pain perception. Sleep Med Rev 2006;10:357-69.

11. Feldman HA, Goldstein I, Hatzichristou DG, et al. Impotence and its medical and psychosocial correlates: results of the Massachusetts Male Aging Study. J Urol 1994;151:54-61.

12. Bozorgmehri S, Fink HA, Parimi N, et al. Association of Sleep Disordered Breathing with Erectile Dysfunction in Community Dwelling Older Men. J Urol 2017;197:776-82.

13. Kellesarian SV, Malignaggi VR, Feng C, et al. Association between obstructive sleep apnea and erectile dysfunction: a systematic review and meta-analysis. Int J Impot Res 2018;30:129-40.

14. Melehan KL, Hoyos CM, Hamilton GS, et al. Randomized Trial of CPAP and Vardenafil on Erectile and Arterial Function in Men With Obstructive Sleep Apnea and Erectile Dysfunction. J Clin Endocrinol Metab 2018;103:1601-11.

15. McBride JA, Kohn TP, Rodriguez KM, et al. PD2707 Incidence and characteristics of men at high risk for sleep apnea in a high volume andrology clinic. J Urol 2018;199:e559-60.

16. Pastuszak AW, Moon YM, Scovell J, et al. Poor Sleep Quality Predicts Hypogonadal Symptoms and Sexual Dysfunction in Male Nonstandard Shift Workers. Urology 2017;102:121-5.

17. Sateia MJ. International classification of sleep disordersthird edition: highlights and modifications. Chest 2014;146:1387-94.

18. Seehuus M, Pigeon $W$. The sleep and sex survey: Relationships between sexual function and sleep. J Psychosom Res 2018;112:59-65.

19. Le HH, Salas RME, Gamaldo A, et al. The utility and feasibility of assessing sleep disruption in a men's health clinic using a mobile health platform device: A pilot study. Int J Clin Pract 2018;72. doi: 10.1111/ijcp.12999.

20. Soterio-Pires JH, Hirotsu C, Kim LJ, et al. The interaction between erectile dysfunction complaints and depression in men: a cross-sectional study about sleep, hormones and quality of life. Int J Impot Res 2017;29:70-5.

21. Everaert K, Anderson P, Wood R, et al. Nocturia is more bothersome than daytime LUTS: Results from an Observational, Real-life Practice Database including 8659 European and American LUTS patients. Int J Clin Pract 2018;72:e13091.

22. Irer B, Celikhisar A, Celikhisar H, et al. Evaluation of Sexual Dysfunction, Lower Urinary Tract Symptoms and Quality of Life in Men With Obstructive Sleep Apnea Syndrome and the Efficacy of Continuous Positive Airway Pressure Therapy. Urology 2018;121:86-92.

23. Bates J, Kohn T, Rodriguez K, et al. PD65-08 Poor sleep quality is associated with clinically significant lower urinary tract symptoms. J Urol 2019;201:e1189-90.

24. Kuhn E, Schwarz EI, Bratton DJ, et al. Effects of CPAP and Mandibular Advancement Devices on Health-Related Quality of Life in OSA: A Systematic Review and Metaanalysis. Chest 2017;151:786-94.

25. Fernandez-Pello S, Gil R, Escaf S, et al. Lower urinary tract symptoms and obstructive sleep apnea syndrome: Urodynamic evolution before and after one year of treatment with continuous positive airway pressure. Actas Urol Esp 2019;43:371-7.

26. Metta RV, Zaka A, Lee VC, et al. Continuous Positive Airway Pressure Adherence In Patients with Obstructive Sleep Apnea \& Symptomatic BPH. Lung 2017;195:255-61.

27. Scovell JM, Pastuszak AW, Slawin J, et al. Impaired Sleep Quality is Associated With More Significant Lower Urinary Tract Symptoms in Male Shift Workers. Urology 2017;99:197-202. 
28. Sigalos JT, Kohn TP, Cartagenova L, et al. Shift Workers With Shift Work Disorder Have Worse Lower Urinary Tract Symptoms. Urology 2019;128:66-70.

29. Shimizu N, Nozawa M, Sugimoto K, et al. Therapeutic efficacy and anti-inflammatory effect of ramelteon in patients with insomnia associated with lower urinary tract symptoms. Res Rep Urol 2013;5:113-9.

30. Mulhall JP, Trost LW, Brannigan RE, et al. Evaluation and Management of Testosterone Deficiency: AUA Guideline. J Urol 2018;200:423-32.

31. Kirby W, Balasubramanian A, Santiago J, et al. MP9106 increased risk of HYPOGONADAL symptoms in shift workers with shift work sleep disorder. J Urol 2017;197:e1220.

32. Wittert $G$. The relationship between sleep disorders and testosterone in men. Asian J Androl 2014;16:262-5.

33. Morley JE, Charlton E, Patrick P, et al. Validation of a screening questionnaire for androgen deficiency in aging males. Metabolism 2000;49:1239-42.

34. Charlier CM, Barr ML, Colby SE, et al. Correlations of Self-Reported Androgen Deficiency in Ageing Males (ADAM) with Stress and Sleep among Young Adult Males. Healthcare (Basel) 2018. doi: 10.3390/healthcare6040121.

35. Levine H, Jorgensen N, Martino-Andrade A, et al. Temporal trends in sperm count: a systematic review and meta-regression analysis. Hum Reprod Update 2017;23:646-59.

36. Swan SH, Elkin EP, Fenster L. The question of declining sperm density revisited: an analysis of 101 studies published 1934-1996. Environ Health Perspect 2000;108:961-6.

37. Sengupta P, Nwagha U, Dutta S, et al. Evidence for decreasing sperm count in African population from 1965 to 2015. Afr Health Sci 2017;17:418-27.

38. Wise LA, Rothman KJ, Wesselink AK, et al. Male sleep duration and fecundability in a North American preconception cohort study. Fertil Steril 2018;109:453-9.

39. Kohn TP, Pastuszak AW. Shift work is associated with altered semen parameters in infertile men. Fertility and Sterility 2017;108:e323-4.

40. Chen Q, Yang H, Zhou N, et al. Inverse U-shaped Association between Sleep Duration and Semen Quality: Longitudinal Observational Study (MARHCS) in

Cite this article as: Kohn TP, Kohn JR, Haney NM, Pastuszak AW, Lipshultz LI. The effect of sleep on men's health. Transl Androl Urol 2020;9(Suppl 2):S178-S185. doi: 10.21037/ tau.2019.11.07
Chongqing, China. Sleep 2016;39:79-86.

41. Jensen TK, Andersson AM, Skakkebaek NE, et al. Association of sleep disturbances with reduced semen quality: a cross-sectional study among 953 healthy young Danish men. Am J Epidemiol 2013;177:1027-37.

42. Palnitkar G, Phillips CL, Hoyos CM, et al. Linking sleep disturbance to idiopathic male infertility. Sleep Med Rev 2018;42:149-59.

43. Rossi SP, Windschuettl S, Matzkin ME, et al. Melatonin in testes of infertile men: evidence for anti-proliferative and anti-oxidant effects on local macrophage and mast cell populations. Andrology 2014;2:436-49.

44. El-Helaly M, Awadalla N, Mansour M, et al. Workplace exposures and male infertility - a case-control study. Int J Occup Med Environ Health 2010;23:331-8.

45. Bisanti L, Olsen J, Basso O, et al. Shift work and subfecundity: a European multicenter study. European Study Group on Infertility and Subfecundity. J Occup Environ Med 1996;38:352-8.

46. Irish LA, Kline CE, Gunn HE, et al. The role of sleep hygiene in promoting public health: A review of empirical evidence. Sleep Med Rev 2015;22:23-36.

47. Kohn TP, Rodriguez KM, Sigalos JT, et al. PD27-08 Poor sleep quality is associated with clinically significant erectile dysfunction. J Urol 2018;199:e560.

48. Chung F, Abdullah HR, Liao P. STOP-Bang Questionnaire: A Practical Approach to Screen for Obstructive Sleep Apnea. Chest 2016;149:631-8.

49. Walia AS, Lomeli LJM, Jiang P, et al. Patients presenting to a Men's Health clinic are at higher risk for depression, insomnia, and sleep apnea. Int J Impot Res 2019;31:39-45.

50. Buysse DJ, Reynolds CF, 3rd, Monk TH, et al. The Pittsburgh Sleep Quality Index: a new instrument for psychiatric practice and research. Psychiatry Res 1989;28:193-213.

51. Chung KF, Lee CT, Yeung WF, et al. Sleep hygiene education as a treatment of insomnia: a systematic review and meta-analysis. Fam Pract 2018;35:365-75.

52. Kuhn E, Weiss BJ, Taylor KL, et al. CBT-I Coach: A Description and Clinician Perceptions of a Mobile App for Cognitive Behavioral Therapy for Insomnia. J Clin Sleep Med 2016;12:597-606. 